\title{
Testing of a New Electricity-Generating TMD by Shaking Table
}

\author{
Dongho Lee*, Dong-ho Ha, Jinkyo F. Choo and Minsung Suh \\ Department of Civil Engineering, Konkuk University, 120 Neungdong-ro Gwangjin-gu, Seoul 143-701, \\ Republic of Korea \\ ${ }^{*}$ Corresponding author
}

\begin{abstract}
This paper presents the series of shaking table tests performed on a new electricity-generating Tuned Mass Damper (TMD) called to as Tuned Mass Piezo-Damper (TMPD), which intends to harvest electric power using the vibrational energy of the structure on which it is attached. The tests were conducted to verify the operational and electricity generation efficiency of the TMPD considering various dispositions of the piezocomposite elements that replace the damper part of the TMD. The results are presented and further improvements are discussed.
\end{abstract}

Keywords-piezoelectric element; tuned Mass Piezo-Damper; energy harvesting; shaking table test

\section{INTRODUCTION}

Modern structures are becoming longer, slender and higher thanks to the advances realized in construction technology. However, this situation renders these structures sensitive to external forces and generates undesired vibrations. Especially, bridges are permanently exposed to dynamic loads like wind and traffic that shorten the fatigue lifetime through repeated vibrations.

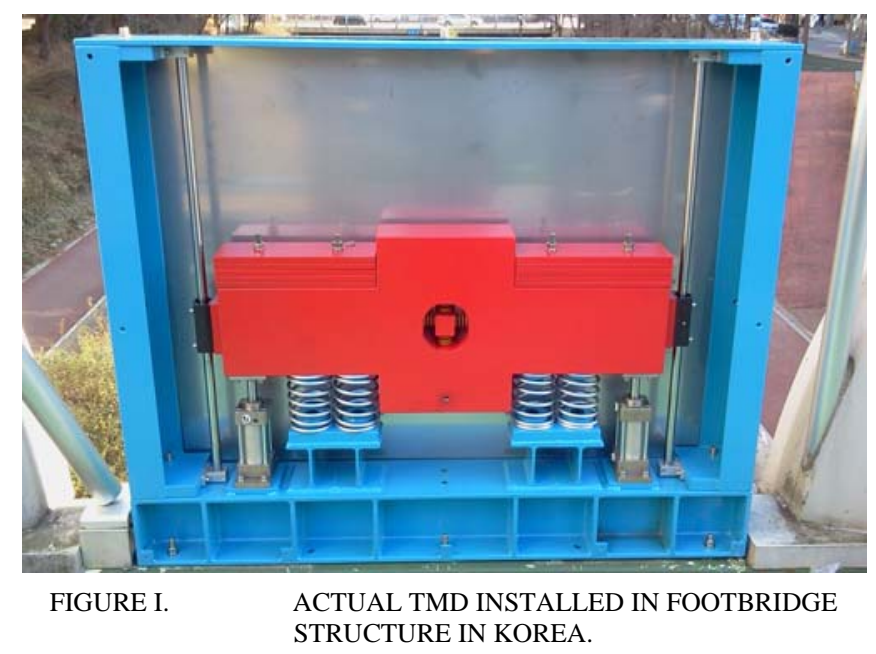

Among the measures taken to reduce these undesired vibrations down to an acceptable level, the Tuned Mass Damper (TMD) is now a common device that has been applied not only in bridges but also in buildings and air traffic control towers. The TMD presents a very simple structure composed of a mass, springs and dampers (Figure 1), of which the damper is the most expensive part. Therefore, our research team in relation with bridge structures developed the Tuned Mass Piezo-Damper (TMPD) shown in Figure 2 as a TMD capable of producing electric power through the replacement of the damper by piezocomposite elements since as mentioned above the bridge is permanently subject to dynamic loading [1-3].

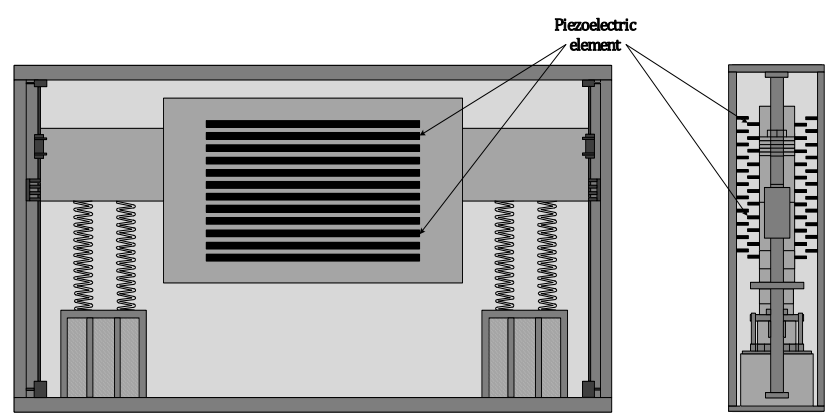

FIGURE II.

CONCEPTUAL DRAWING OF TMPD.

The piezoelectric effect describes the relation between a mechanical stress and an electrical voltage in solids. Concretely, when a mechanical stress is applied on the piezoelectric material, electrical charges appear, and this voltage is proportional to the stress. Such material is generally a piezoceramic but piezoceramic is featured by its extreme brittleness that makes it inappropriate to civil engineering application as it is. Therefore, the piezoceramic is generally combined with other materials to form a piezocomposite $[4,5]$. In the present application, the piezoceramic is combined with and stainless steel plate so as to improve the resistance to shocks and the electricity generating efficiency of the piezoelectric [6].

As shown in Figure 2, several piezocomposite elements are installed in the TMPD. Note that the TMPD is intended to control the vertical vibrations of a fictive footbridge, and by so, to be installed vertically on the fence of the footbridge as shown in Figure 1.

Accordingly, this study presents the series of shaking table tests on TMPD prototypes to verify the electricity generation efficiency of the TMPD considering various dispositions of the piezocomposite elements. 


\section{TEST OF TMPD}

\section{A. Test Using Shaking Table in Horizontal Direction}

A first prototype of the TMPD was designed and fabricated as shown in Figure 3 so as to generate electricity by hitting the piezocomposite element through the motion of the mass since a former study on the piezocomposite element itself [6] reported that the best efficiency was obtained by hitting the element.

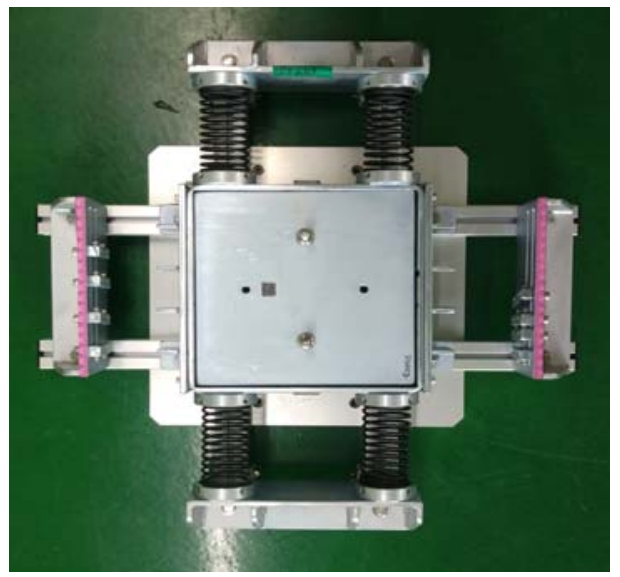

FIGURE III.

FIRST TMPD PROTOTYPE.

The problem here is that the natural frequency of the piezocomposite element is about $60 \mathrm{~Hz}$ but that the pedestrian load is known to run around $2 \mathrm{~Hz}$. In addition, the best electricity-generating efficiency of the piezocomposite element is realized by exciting it near its natural frequency. Accordingly, the first TMPD prototype was conceived by adopting a modular framework to enable various configurations be considered like the possibility to modify the natural frequency of the TMPD by changing the number of mass modules and by changing the stiffness of the springs, the number of hitting plates to vary the frequency of excitation of the piezocomposite element, and the number of piezocomposite elements attached to the system.

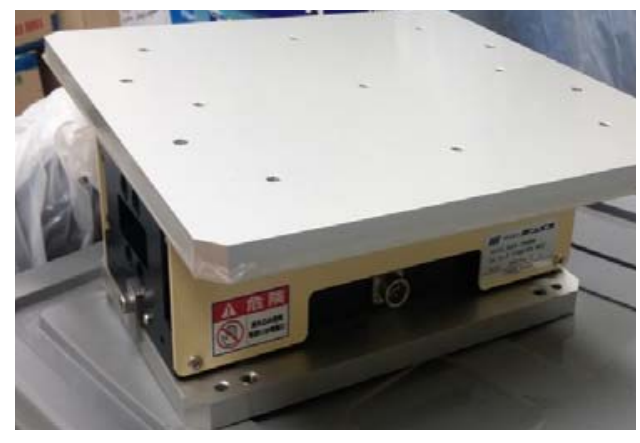

FIGURE IV.

SMALL-SCALE SHAKER.

The test was planned to hit the piezocomposite elements inserted in the TMPD with the highest frequency possible so as to verify its electricity-generating efficiency. The test used the small shaker shown in Figure 4. Due to the characteristics of the shaker, the TMPD was installed horizontally and the motion of the mass was induced horizontally.

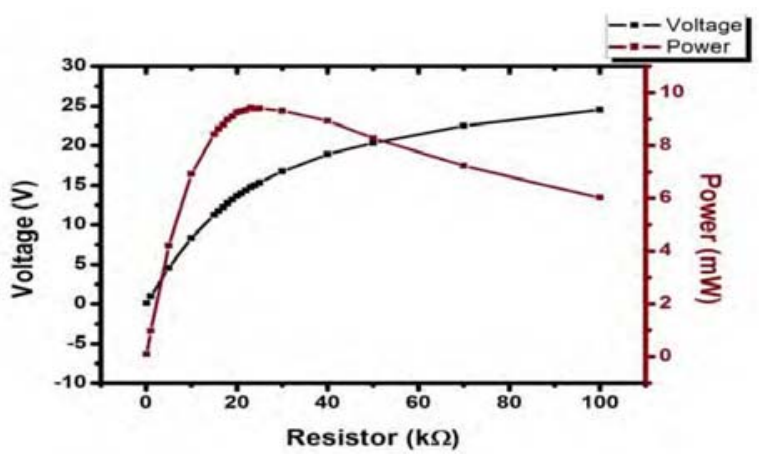

FIGURE V. OUTPUT VOLTAGE AND POWER OF FIRST TMPD PROTOTYPE USING 1 PIEZOCOMPOSITE ELEMENT.

Figure 5 shows the results of the test conducted using one piezoelectric element and by connecting a resistance to the system. It can be seen that relatively large voltage is output despite of the discrepancy between the natural frequencies of the piezoelectric element and the exciting vibration. However, the chosen configuration of the TMPD which hit the piezoelectric element appeared to result in the failure of the piezoceramic as shown in Figure 6.
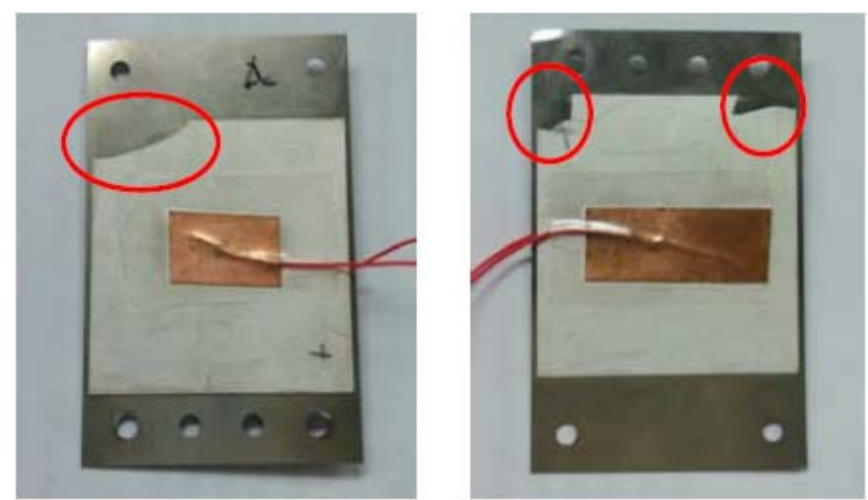

FIGURE VI.

FAILURE PATTERNS OF PIEZOCOMPOSITE ELEMENTS.

This first series of tests indicated the potential of the TMPD for generating electric power using the motion of the mass. However, despite of the clear efficiency of the proposed TMPD, the adoption of another configuration seems to be necessary in term of the durability of the system. Moreover, due to the limitation brought by the small-scale shaker, the present configuration revealed deficiencies in simulating realistically the operational environment of the TMPD like the loss of the inertial force of the mass that would in reality move vertically, and the resulting frictional loss disturbing the free motion of the mass after the end of the excitation.

Based upon these results, a second TMPD prototype was designed and fabricated to simulate realistically the expected operation of the TMPD.

\section{B. Test Using Shaking Table in Vertical Direction}

Figure 7 shows the second TMPD prototype designed and fabricated to solve the problems observed in the test of the first TMPD prototype. 


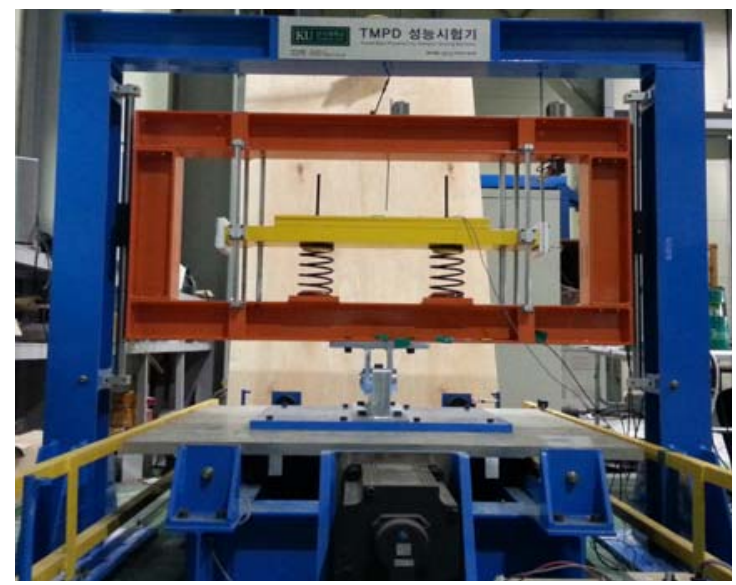

FIGURE VII.
SECOND TMPD PROTOTYPE INSTALLED FOR SHAKING TABLE TEST.

First, the second TMPD prototype was intended to operate vertically. Therefore, the TMPD was disposed in a frame as shown in Figure 8 to make it remain vertical all along the test using the shaking table shown in Figure 9.

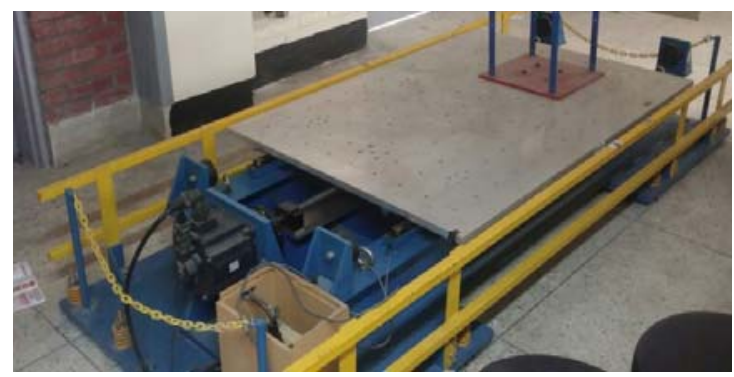

FIGURE VIII

SHAKING TABLE.

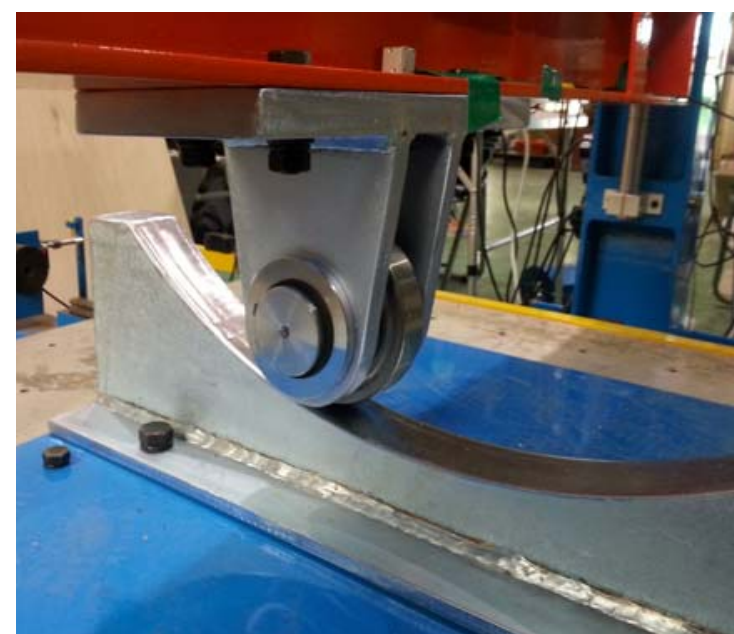

FIGURE IX.

VIEW OF INTERFACE BETWEEN FRAME AND SHAKING TABLE.

This frame equipped with a ball-bearing at its bottom converts the horizontal motion of the shaking table to the vertical motion of the TMPD as shown in Figure 10 by means of a curved guide installed on the shaking table (Figure 9).
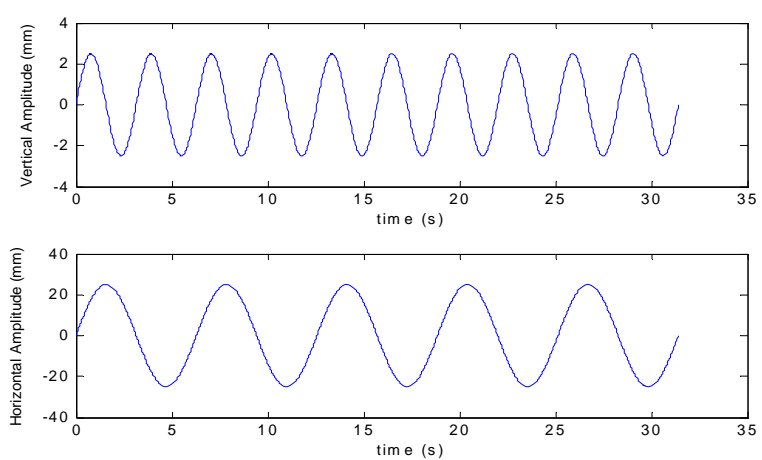

FIGURE $X . \quad$ CONVERSION OF HORIZONTAL MOTION OF SHAKING TABLE (DOWN) INTO VERTICAL MOTION OF FRAME (UP).

The configuration chosen for the test adopted a mass of about $95 \mathrm{~kg}$ to increase the damping ratio. In addition, recalling that the first series of test resulted in the failure of the piezocomposite elements due to shock, the approach here was to induce the deformation of the piezocomposite elements without hitting them but by adopting cantilevered elements that would displace the free end like that of the cantilevered beam under vertical motion of the fixed support. The shaking table test of the second TMPD prototype enabled as designed to excite the TMPD vertically through the horizontal motion of the table. In addition, as shown in Figure 10, the exciting frequency of the shaking table could be doubled in the vertical direction.

Since the test was planned to be performed with an input frequency of $2 \mathrm{~Hz}$. The dimensions of the piezocomposite element were modified so as to decrease as possible its natural frequency. The considered piezocomposite elements are listed in Table 1 with their corresponding natural frequencies. It can be seen that the natural frequency of the piezocomposite element could be reduced by nearly $75 \%$ compared to the original one. Therefore, the use of PZT-B would in some extent counteract the loss of electricity-generating efficiency that will likely occur by changing the method by which the piezocomposite element is excited. Recall that in the first configuration, the element was directly hit whereas the element is indirectly deformed in a cantilevered system in the second configuration.

TABLE I. CHARACTERISTICS OF PIEZOCOMPOSITE ElEMENTS CONSIDERED IN THE VERTICAL SHAKING TABLE TEST.

\begin{tabular}{|c|c|c|c|}
\hline \multirow[b]{2}{*}{ Element } & \multicolumn{2}{|c|}{ Dimensions } & \multirow[b]{2}{*}{$\begin{array}{c}\text { Natural } \\
\text { Frequency } \\
\text { (Hz) }\end{array}$} \\
\hline & $\begin{array}{c}\text { Piezoceramic } \\
\left(\mathrm{mm}^{2}\right)\end{array}$ & $\begin{array}{c}\text { Stainless steel sub- } \\
\text { plate } \\
\left(\mathrm{mm}^{2}\right)\end{array}$ & \\
\hline PZT-A & $38 \times 38$ & $40 \times 60$ & 61.29 \\
\hline PZT-B & $38 \times 38$ & $40 \times 120$ & 15.77 \\
\hline
\end{tabular}

Figures 11 and 12 plot the results of the tests using PZT-A and PZT-B, respectively. It can be seen that the peaks occur around $2 \mathrm{~Hz}$ because the TMPD was designed to have natural frequency of $2 \mathrm{~Hz}$. This means that the mass will experience the largest displacement under excitation of $2 \mathrm{~Hz}$. As a matter of fact the output power is directly correlated to the output voltage. 
In concern with the presented graphs, the corresponding RMS power is calculated to be nearly $0 \mathrm{Wh}$ for PZT-A and 0.0684 Wh for PZT-B. These values account only for a single piezoelectric element attached to the TMPD and are given for information. Nevertheless, these values show that the selected configuration enables to excite the piezoelectric element enough to generate electricity and that a slight change in the dimensions of the element can improve the efficiency according to the purposed application.

Finally, the configuration chosen for the second TMPD prototype prevented the failure of the piezoelectric element, which is highly appreciable in term of durability.
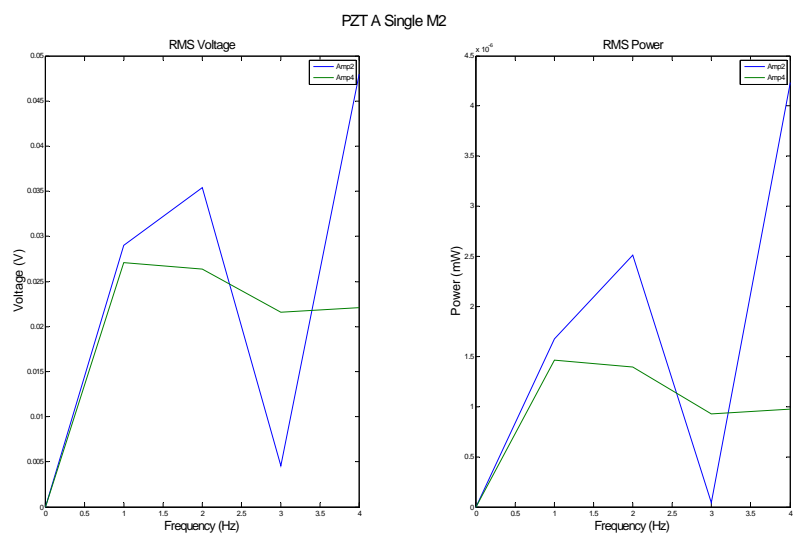

FIGURE XI. VOLTAGE AND POWER OUTPUT OF PZT-A.

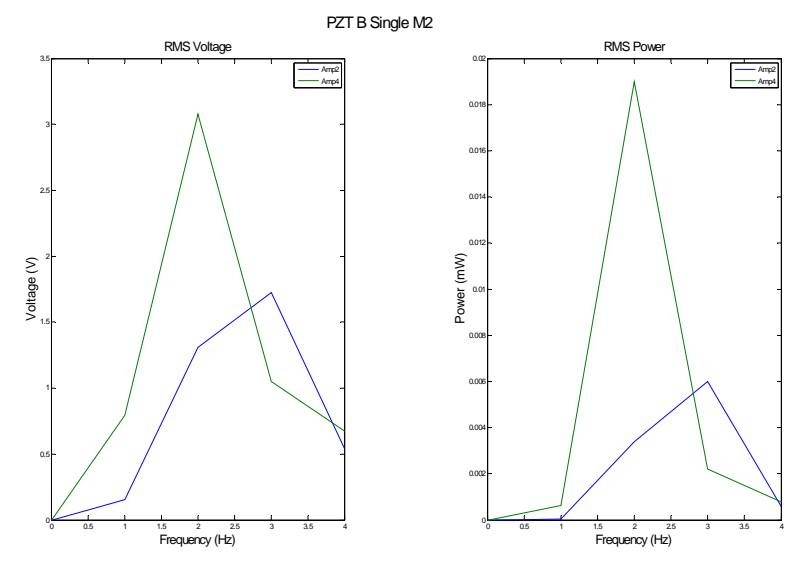

FIGURE XII. VOLTAGE AND POWER OUTPUT OF PZT-B.

\section{CONCLUSIONS}

This paper introduced a new type of TMD capable of producing electric power through the replacement of the damper by piezocomposite elements. This so-called TMPD was tested in order to examine its efficiency in generating electric power. To that goal, a first TMPD prototype was designed, fabricated and subject to horizontal shaking test using a small-scale shaker. The experimental results demonstrated the feasibility of the system but also revealed several problems like the lack of realism of the testing setup and the failure of the piezocomposite elements subject to direct hitting.
Accordingly, a second TMPD prototype was designed to overcome the problems pointed out in the first series of test. In the second series of test, a frame was conceived to transform the horizontal motion of the shaking table into a vertical motion, and the TMPD was configured to deform indirectly the piezocomposite elements in a cantilevered way without hitting them. The experimental results showed also the feasibility of the system but also a certain loss of electricitygenerating efficiency due to the adopted configuration.

Further studies will consider the use of PVDF (PolyVinylidene fluoride) instead of the piezoceramic-based composite element since it is known that PVDF exhibits optimal efficiency under large deformation.

\section{ACKNOWLEDGMENTS}

This work is financially supported by Korea Minister of Ministry of Land, Infrastructure, and Transport (MOLIT) as U-City Manpower Development Program.

This work was supported by the *Energy Efficiency \& Resources Core Technology Program of the Korea Institute of Energy Technology Evaluation and Planning (KETEP), granted financial resource from the Ministry of Trade, Industry \& Energy, Republic of Korea. (NO. 20142020103970).

\section{REFERENCES}

[1] M. Suh, D. H. Ha, J. F. Choo, H. Han, and D. Lee, "Development of test prototype for electricity-generation tuned-mass-damper,” International Conference on Electronics, Computer and Information Technology, pp.153-157, 2016

[2] M. Suh, D. H. Ha, J. F. Choo, S. G. Chang and, D. Lee "Development of vertical excitor system by using horizontal shaking table", Earthquake Engineering Society of Korea, vol. 20, pp. 61-62, 2016.

[3] M. Suh, "Experimental study on tuned mass piezoelectric damper using shaking table,” M.Sc Dissertation, Dept. of Civil Engineering, Konkuk University, 2016. (in Korean)

[4] J. Zhao, N. S. Goo, and D. H. Ha, "Design and performance evaluation of a $\mathrm{d}_{33}$-mode piezocomposite electricity generating element,” J. Mech. Sci. Technol., vol. 28(1), pp. 15-23, 2014.

[5] V. L. Pham, N. S. Ha, N. S. Goo, and J. F. Choo, "Effects of cyclic impacts on the performance of a piezo-composite electricity generating element in a $\mathrm{d}_{33}$-mode energy harvesting," J. Nanosci. Nanotechnol., vol. 14(10), pp. 7410-7418, 2014.

[6] D. Song, M. S. Woo, J. H. Ahn, S. K. Hong, S. B. Kim, and T. H. Sung, "Study on improving current generating time of piezoelectric energy harvesting system,” J. Electroceram., DOI 10.1007/s10832-014-9974-5, 2014 